\title{
Titel/Title: Die Anlagefonds und der Mittelstand: Paul Windolfs und Christoph Deutschmanns Studien über den „Finanzmarkt-Kapitalismus“
}

Autor*innen/Author(s): Uwe Schimank

Veröffentlichungsversion/Published version: Postprint

Zeitschriftenartikel/Journal article

\section{Empfohlene Zitierung/Recommended citation:}

Schimank, Uwe (2007): Die Anlagefonds und der Mittelstand: Paul Windolfs und Christoph Deutschmanns Studien über den „Finanzmarkt-Kapitalismus“. In: Leviathan, (35), 47-61.

Verfügbar unter/Available at:

(wenn vorhanden, bitte den DOI angeben/please provide the DOI if available)

This is a post-peer-review, pre-copyedit version of an article published in Leviathan. The final authenticated version is available online at: https://doi.org/10.1007/s11578-007-0005-7

Zusätzliche Informationen/Additional information:

Der Autor kann kontaktiert werden unter: uwe.schimank@uni-bremen.de 


\section{Uwe Schimank}

Die Anlagefonds und der Mittelstand: Paul Windolfs und Christoph Deutschmanns Studien über den „Finanzmarkt-Kapitalismus“

\section{Einleitung}

Kluge Sozialwissenschaftler haben mit Romanschriftstellern, Theaterautoren, Filmemachern und manch einem Historiker etwas gemeinsam - die Freude am überraschenden Plot, den Spaß am Wiedererkennen hintergründiger Verlaufsfiguren des Geschehens im Chaos der Ereignisse. Die Geschichte des modernen Kapitalismus ist ein solcher Stoff, der zahllose Drehbücher inspiriert hat; und besonders für Gesellschaftstheoretiker blieb die dramaturgische Würdigung der kapitalistischen Entwicklung immer von hohem Reiz.

„Denn sie wissen nicht, was sie tun!“ Das ist eine nachdenklich stimmende Denkfigur, die Spannung verspricht und soziologischer Aufklärung auf der Linie von Robert K. Mertons (1936) „unanticipated consequences of purposive social action“ zur Ehre gereicht. Es geht dabei um Akteure, die Effekte auslösen, an die sie nie gedacht haben und die sie höchst unerwünscht finden; es geht um unerwünschte Wirkungen, die nicht irgend jemanden treffen, sondern auf ihre Urheber selbst zu- rückfallen; es geht um Selbstschädigungen bis hin zu Katastrophen, die nicht auf freie Entscheidungen zurückgehen, sondern auf strukturelle Zwänge des sozialen Zusammenwirkens. In dieser dreischrittartigen Steigerung lässt sich das Drama des Kapitalismus wohl inszenieren - gestern wie heute. Der Klassiker - Karl Marx - ist bekannt, wenngleich er oft missverstanden wurde, weshalb er hier anfangs kurz in Erinnerung gerufen wird. Das Hauptaugenmerk gilt aber den zeitgenössischen Dramatikern des „Finanzmarkt-Kapitalismus": Wie setzen sie die wohlbekannte Denkfigur der krisenhaften Dynamik des Kapitalismus neu in Szene? Die Rede ist von Paul Windolf und Christoph Deutschmann, die jüngst mit einander ergänzenden Überlegungen hierzu hervorgetreten sind.

Die Würdigung ihrer dramaturgischen Leistung erfolgt hier wohlgemerkt nicht als Abgleich mit dem Realgeschehen, sondern „theorieästhetisch“: Wie erfindungs- reich wird die Denkfigur inszeniert? Manche mögen diesen schöngeistigen Beurteilungsmassstab mit szientifischem Furor abtun; aber jene Leser, die mehr Erfahrung im Geschäft der Wissenschaft mitbringen, werden behutsamer urteilen und wissen 
$(\leftarrow$ p.47) wollen, was denn von Windolf und Deutschmann - neben empirischen Daten - als Theoriearchitektur präsentiert wird. Hinter diesem Interesse steckt nicht zuletzt die Einschätzung, dass theoretische Raffinesse die empirische Arbeit mehr inspiriert als biedere Hausmannskost - und dass gute Empiriker theoretische Feinschmecker sein sollten.

\section{Dieklassische Tragödie: Selbstabschaffung der Kapitalisten}

Dass der Kapitalismus am Antagonismus von Kapitalisten und Arbeiterklasse unter- gehen werde, ist eine vordergründige Lesart der Diagnose, die Marx dem Kapitalismus des 19. Jahrhunderts gestellt hatte. Es ist richtig: Den Arbeitern, die ohne Besitz an Produktionsmitteln nur ihre Arbeitskraft zu verkaufen hatten, standen leibhaftige Kapitalisten gegenüber, die die Zwangslage der Arbeiter ausbeuteten; letztere würden - so die revolutionäre Vision - aus nacktem Eigeninteresse langfristig dazu getrieben, den kollektiven Klassenkampf gegen ihre Ausbeuter zu führen, und ihn sieg- reich ein für alle Mal beenden. Die Arbeiter hatten schließlich nichts zu verlieren

„als ihre Ketten“. Die Kapitalisten würden immer mehr Menschen immer heftiger gegen sich aufbringen, die Ausbeutung sozusagen übertreiben - freilich nicht aus freien Stücken, purer Bosheit oder persönlicher Profitgier, sondern weil sie dem unbarmherzigen strukturellen Zwang der Konkurrenz untereinander unterliegen, der

sie zur Profitakkumulation antreibt. ${ }^{1}$ In dieser Konkurrenz zu bestehen und weiter

im Rennen zu bleiben oder selbst der Produktionsmittel verlustig zu gehen und proletarisiert zu werdenso stellte sich für Marx die Lage der Kapitalisten dar. Was wie ein Kampf zweier Klassen aussieht, ist also, genauer besehen, die Selbstabschaffung einer der beiden Klassen. Die andere Klasse stellt in diesem Geschehen nur den herbeigerufenen Sterbehelfer dar: Die Bourgeoisie ,[...] produziert [...] ihren eignen Totengräber.“ (Marx/Engels 1848, S. 57) Dieses transintentionale Tun macht die Kapitalisten zum historischen Subjekt. Denn mit ihrer Selbstabschaffung als Klasse schaffen sie zugleich den Kapitalismus als Gesellschaftsformation ab.

Ein zweiter von Marx herausgearbeiteter Zusammenhang läuft parallel auf das Gleiche hinaus. Seiner Arbeitswertlehre gemäß kann nur „lebendige Arbeit“ den Mehrwert hecken. Doch die Kapitalisten betreiben in der „großen Industrie“ (Marx 1867, S. 391-530) die immer weiter voranschreitende Substitution der menschlichen Arbeitskraft durch Maschinen. Ganz abgesehen von der - nicht unwichtigen Frage, wer dann noch das Geld verdient, mit dem die so produzierten Waren gekauft werden können, was

${ }^{1}$ Kartellbildung und Monopolisierung - von Marx behandelt - sind nichts als Zwischenstadien dieser 
die Voraussetzung für die Realisierung von Profiten ist: Schon einen Schritt zuvor kommt die Mehrwertproduktion in der „menschenleeren Fabrik“ (Brödner 1985) endgültig zum Erliegen. In dem herbeigeführten „tendenziellen Fall der Profitrate“ (Marx 1894, S. 221-277), gekoppelt mit einer chronisch werden- $(\leftarrow$ p.48) den Überproduktionskrise, erledigen sich die Kapitalisten ebenfalls selbst - dieses Mal mittels der Technik, deren unaufhaltsamer Fortschritt für Marx ebenso unbezweifelbar war wie der revolutionäre Elan der Arbeiterklasse.

Das alles kam bekanntlich ganz anders, und den Kapitalismus gibt es heute noch. Zum einen ist die Arbeitswertlehre falsch, der Profit hat andere Ursprünge, als Marx dachte. ${ }^{2}$ Zum anderen hat die Ausbeutung der Arbeiter nicht zu jenem Grad der Verelendung geführt, der die Revolution einläutet, sondern - in Kombination mit

der Ausplünderung der globalen „Peripherie“ - zum hiesigen Sozialstaat; und dieser hat in Kombination mit starken Gewerkschaften dafür gesorgt, dass in Europa und Nordamerika eine breite Mitte der Gesellschaft entstand, die über genügend Kauf- kraft verfügt, um einer wachsenden Wirtschaft zu Profiten zu verhelfen. Eduard Heimann (1929) zeichnete schon in der Weimarer Republik ein Kapitalismusbild, in dem die gewerkschaftlich und sozialdemokratisch organisierte Arbeiterschaft gerade nicht das Werkzeug der Selbstabschaffung der Kapitalisten abgibt, sondern ihren sozialpolitischen Schutzengel - freilich, anders als Engel sonst, durchaus aus handfestem Eigeninteresse.

„Saving capitalism from itself“ : ${ }^{3}$ In der seit den 1950er Jahren aufkommenden

„affluent society“ (Galbraith 1958) war diese Deutung der Arbeiterschaft und erst recht der zahlenmäBig ganz entgegen der Prognose von Marx erstarkenden Mittelschicht so in Fleisch und Blut übergegangen, dass der Kapitalismus - sowohl fak- tisch als auch in dem Bild, das die Gesellschaftstheoretiker von ihm zeichneten - geradezu in harmonischer Selbstzufriedenheit zu ruhen schien. Wer weiter an den Klassenkampf glaubte, war ein Ewiggestriger. An der „Kritischen Theorie“lässt sich exemplarisch vorführen, welche theoriepolitischen Optionen dann noch blieben. Entweder man verzweifelte im „Grandhotel Abgrund“" an der Unbesiegbarkeit des kapitalistischen Verhängnisses, wie Georg Lukacs über Theodor W. Adorno und Max Horkheimer spottete; oder man schöpfte verzweifelte revolutionäre Hoffnung aus Randgruppen und Studentenprotesten, wie es Herbert Marcuse (1964) tat. Letzteres erwies sich schnell als unplausibel.

\footnotetext{
${ }^{2}$ Hier ist nicht der Ort, darüber zu debattieren, woher Profite stammen. Siehe als Theorie- angebot zur Rettung der Arbeitswertlehre neuerdings Deutschmanns (2005, S. 72 f.) an Joseph Schumpeter orientierte Idee, ,außerordentliche Arbeit“, die Neues in die Welt set- ze, sei die Quelle unternehmerischer Profite.
}

${ }^{3}$ Eine Formulierung von Gøsta Esping-Andersen - siehe dazu Klundt (2005). 
Wenn wir diverse Zwischenschritte überspringen, leben wir heute im „Finanzmarkt- Kapitalismus“. Windolf (2005a; 2005b) und Deutschmann (2005; 2006a) tragen hierzu zahlreiche empirische Befunde und theoretische Konzepte zusammen und $(\leftarrow \mathbf{p . 4 9})$ verdichten sie zu einem höchst eindrucksvollen Bild. Denkt man die von Windolf und Deutschmann vorgestellten Strukturdynamiken des gegenwärtigen Kapitalismus zu Ende, kommt man abermals zu einer Selbstabschaffungs-Diagnose des Kapitalismus. Doch das historische Subjekt der Abschaffung sind diesmal nicht die Kapitalisten im Sinne einer durch den Besitz an Produktionsmitteln herrschenden Klasse. Im „Finanzmarkt-Kapitalismus“ schafft sich vielmehr die den sozialpolitisch gezähmten Kapitalismus der „,affluent society“ tragende gesellschaftliche Mitte selbst ab - vom Facharbeiter, Büro- und Verwaltungsangestellten und Kleinunternehmer bis hin zum Professor oder niedergelassenen Arzt.

Diese von Windolf und Deutschmann noch nicht explizit formulierte brisante Quintessenz ihrer Überlegungen könnte eine - dramaturgisch betrachtet - geradezu geniale Ironie der Geschichte sein: Genau jene sozialen Gruppen, die laut Marx als Erste von der historischen Bühne verschwinden sollten, um gewissermaßen den bei- den Hauptakteuren beim Showdown nicht im Wege zu stehen, und die dann ganz entgegen von Marx' Regieanweisung die Hauptrolle übernahmen und das Schau- spiel erst einmal und vermeintlich auf Dauer in Richtung Happy End umschrieben, könnten letzten Endes doch noch eine unbeirrbare Neigung fürs tragische Fachentwickeln. Mit anderen Worten: Wie vormals die Kapitalisten könnte die gesellschaftliche Mitte als historisches Subjekt auftreten, das eine spezifische Ära des Kapitalismus - hier: des Wohlfahrtskapitalismus - zunächst getragen hat, um sie sodann ins Grab zu legen. Schon Ende der 1970er Jahre wurde Deutschland wissenschaftlich und publizistisch als „Zweidrittel-Gesellschaft" etikettiert. Das verwies auf eine nicht länger bagatellisierbare Anzahl von Gesellschaftsmitgliedern, die - wie es gut zehn Jahre später hieß - Opfer gesellschaftlicher „Exklusion“ waren (Kronauer 1998). Was Niklas Luhmann (1994) erst in der Dritten Welt und in Randzonen der Ersten Welt entdecken musste, war genauer hinschauenden Beobachtern längst als ein Phänomen bekannt, auf das man auch in vielen Regionen der fortgeschrittensten westlichen Länder gleich um die Ecke stößt. Pierre Bourdieu und seine Forschergruppe dokumentierten - so der berühmte Buchtitel (1993) - „das Elend der Welt", also die Lage de-

rer, die an den gesellschaftlichen Rand gedrängt worden waren. ${ }^{4}$ Dies war aufrüttelnd genug - jedoch kennt man das „Lumpenproletariat“ und andere Gruppen von „Unterklassen“ (Dahrendorf 1992) und „Überflüssigen“ (Bude 1998) schon seit Anbeginn der kapitalistischen Gesellschaft. Selbst wenn der weltgesellschaftliche Maßstab die Größenverhältnisse umkehrt und die Exklusion nicht mehr nur eine

\footnotetext{
${ }^{4}$ Siehe auch die parallele Untersuchung von Schultheis/Schulz (2005) zu Deutschland.
} 
Randgruppe, sondern die Mehrheit der Weltbevölkerung trifft, stört das ja - jenseits weihnachtlicher Spenden für „Brot für die Welt“ - zunächst einmal niemanden, der in den westlichen Ländern lebt und sein Auskommen hat. Wirklich beunruhigend wurde es erst, als die gesellschaftliche Mitte hierzulande eine zunehmende „Prekarität“ (Bourdieu 1998) ihrer Lage erfuhr. $(\leftarrow \mathbf{p . 5 0})$

Inzwischen kursiert das Wort vom „Prekariat“ in der öffentlichen Debatte (DIE ZEIT 10/2006) und bezieht sich gerade auch auf gut ausgebildete Söhne und Töchter aus Mittelschichtfamilien, die sich nach ihrem Studium von einem Praktikum zum nächsten Projekt hangeln und heute nicht wissen, was morgen kommt; oder auf Facharbeiter Ende Vierzig, die nicht mehr sicher sein können, dass ihr Betrieb - der zuverlässig Gewinne macht - nicht von einem Investor übernommen werden wird, und was dann mit ihrem Arbeitsplatz geschieht. Die „Generation Praktikum“ (DIE ZEIT 14/2005; DER SPIEGEL 31/2006) lebt aus, was Douglas Coupland (1991) als „Generation X“ bereits Anfang der 1990er Jahre literarisch und treffsicher geschildert hat; und um das Bild zu vervollständigen, muss hinzugefügt werden, dass inzwischen nicht wenige Eltern dieser Generation erfahren müssen, dass auch sie nicht gegen sozialen Abstieg gefeit sind. Die gesellschaftliche Mitte, ob jung oder alt, steht heutzutage unter einem ungeheuren und immer noch wachsenden Druck. Was Marx für den Mittelstand seiner Zeit lapidar konstatierte, was dann aber nicht ein- trat, steht heute wieder als Menetekel im Raum: die Abschaffung der Mitte. Anders als Marx es damals sah, wäre die Mitte heute allerdings kein Opfer anderer Kräfte, sondern legte selbst Hand an sich - und nähme damit zugleich entschlossen, wenngleich blind das Heft des kapitalistischen Geschehens in die Hand.

Die einzelnen Bauelemente des komplizierten Mechanismus dieser transintentionalen Selbstabschaffung tragen Windolf und Deutschmann in ihren Charakterisierungen des heutigen „Finanzmarkt-Kapitalismus“ zusammen. „Finanzmarkt-Kapitalismus“ bedeutet zunächst, dass sich die Finanzierung großer Unternehmen von den Kredite gebenden Banken auf Aktienbeteiligungen durch Investment-Fonds einschließlich der Renten- und Immobilien-Fonds - verlagert hat. Unternehmen, die für ihr Operieren und ihre weitere Expansion zusätzliches Kapital benötigen, beschaffen es sich zunehmend nicht mehr als Kredit von einer Hausbank, sondern durch Aktienemission. Hinter diesem Wandel der Unternehmensfinanzierung, der sich seit den 1970er Jahren zunächst in den USA vollzog und in Deutschland erst in den letzten Jahren deutlicher erkennbar geworden ist, steckt ein Bündel ineinander- greifender Ursachen:

Weltweit ist ił der „affluent society“ immer mehr anlagebereites Kapital verfüg- bar geworden, dessen Eigentümer sich nicht mit den mageren Zinssätzen der Banken zufriedengeben wollen - und auch nicht können, wie noch deutlich wer- den wird. Es ist ,[...] das langanhaltende, nunmehr seit fast sechzig Jahren durch keine großen Kriege und Inflationen mehr unterbrochene Wachstum derprivaten Finanzvermögen [...]“ (Deutschmann 2005, S. 80), das die Dynamik in Richtung „Finanzmarkt-Kapitalismus“ in 
Bewegung gesetzt hat und weiter in Bewegung hält. Ein „,chronischer Kapitalüberfluß“ (Deutschmann 2006a, S. 11) dringt auf seine Verwertung. ${ }^{5}(\leftarrow$ p.51)

Die Fondsgesellschaften, die dieses Kapital durch das Versprechen höherer Rendite bündeln, haben den Banken den Markt der Unternehmensfinanzierung zu- nehmend streitig gemacht. Das hat dazu geführt, dass die Banken sich Schritt für Schritt aus dem Kreditgeschäft herausgezogen haben und statt dessen quasi eine „Identifikation mit dem Angreifer“ - selbst schwerpunktmäßig zu Investmentbanken geworden sind. Die Deutsche Bank ist hierzulande nur das prominenteste Beispiel für diesen grundlegenden Strategiewechsel.

Diese Vorgänge wurden zum einen dadurch ermöglicht, dass die nationalstaatlichen Einhegungen von Märkten - insbesondere von Finanzmärkten - nicht zuletzt auf Druck der Investmentfonds abgebaut wurden und die Globalisierung des wirtschaftlichen Geschehens rasch voranschreiten konnte. Zum anderen schufen die elektronischen Informations- und Kommunikationstechnologien die Möglichkeit weltweiter Echtzeit-Transaktionen, die das Geschehen auf den Finanzmärkten rasant beschleunigt haben.

Die insbesondere im „Rheinischen Kapitalismus“ ehemals etablierte Vormachtstellung der großen Banken hatte den kreditfinanzierten Unternehmen eine verlässliche Kapitalbasis verschafft und ihnen - auch durch die Kontrollrechte der Bankenvertreter in den Aufsichtsgremien - eine spezifische Logik der Profiterzielung auferlegt. Ein Kreditgeber ist an der langfristigen Rückzahlungsfähigkeit seines Kreditnehmers interessiert und will nicht, dass dieser riskante Strategien der Profitmaximierung wagt; denn der Kreditgeber partizipiert ja auch im Erfolgsfall nicht an den hohen Profiten. Andersherum: Eine kreditgebende Bank hält ein kreditnehmendes Unter- nehmen zu sicheren Profiten mittlerer Höhe an. Erfolgsausweis des Unternehmens und seiner Manager ist unter diesen Bedingungen nicht die Höhe des Profits, sondern das „organische“ Wachstum der Organisation: Je größer, desto besser. Da dieses Wachstum immer wieder neue Kredite erforderlich macht, dient es auch den Interessen der Banken.

Dieses sich selbst reproduzierende System des Gebens und Nehmens zwischen Banken und Großunternehmen ist durch das Eindringen der Investment-Fonds in die Finanzmärkte weitgehend zerstört worden, ${ }^{6}$ und die Logik der unternehmerischen $(\leftarrow \mathbf{p . 5 2})$ Profiterzielung hat sich zur Maximierung des

\footnotetext{
${ }^{5}$ Deutschmann (2005, S. 81) sieht hierin sogar den ,[...] tieferliegenden gesellschaftlichen Prozess [...], der auch ohne [...]“ das Mitwirken der Fonds zu einer krisenhaften Zuspitzung führt: die zunehmende Knappheit an Investionsmöglichkeiten für immer mehr in- vestitionsbereites Kapital. Hier wäre freilich zu fragen, ob sich diese Problematik - außer durch Kapital vernichtende Kriege, gegen die auch die Bevölkerung entwickelter westlicher Länder nicht gefeit ist - nicht durch die großen neuen Investitionsmöglichkeiten in Ländern wie China und Indien entschärft. Und bis auch dort kein Kapital mehr benötigt wird, steht vermutlich die Besiedlung des Weltraums als riesiges Investitionsfeld an - will sagen: Nicht nur die von Deutschmann in Anlehnung an Schumpeter betonte und seiner Einschätzung nach an Grenzen stoßende Dynamik ,,schöpferischer Zerstörung“" von Produkten, sondern vielleicht mehr noch eine Dynamik schöpferischer Erschließung von Bedarf könnte auch weiterhin Investitionschancen bereitstellen.

${ }^{6}$ Das spiegelt sich eindrucksvoll darin wider, dass sich in Deutschland die Netzwerke der Unternehmensverflechtungen, die durch die großen Banken zusammengehalten werden, in den letzten Jahren rapide ausgedünnt haben (Höpner/Krempel
} 
shareholder value gewandelt. Fonds investieren per Aktienbeteiligung viel kurzfristiger in ein Unternehmen, als es die Banken getan haben; Fonds sind an einer schnellen Profitmaximierung in Gestalt eines steigenden Aktienkurses interessiert, um die Aktien im richtigen Moment eher früher als später - profitabel zu veräußern und das disponibel gewordene Kapital in einem anderen Unternehmen anzulegen. Die Manager der Unternehmen werden diesem Interesse der Fonds teils durch deren Vertreter in den Aufsichtsgremien und Aktionärsversammlungen unterworfen, wobei die Fonds flankierend auch auf der politischen Bühne für eine Stärkung von Aktionärsrechten gesorgt haben - dabei im Interesse der Kleinaktionäre auftretend, weil sie selbst oft auch nur geringe Anteile an einem Unternehmen halten. Teils werden die Manager aber auch durch Aktienoptionen zu Teilhabern am Gewinn der Investment-Fonds gemacht und brauchen sich dann gegebenenfalls nicht einmal solchen feindlichen Übernahmen entgegen stemmen, die sie ihren Job kosten - der goldene Handschlag durch den Verkauf der Aktien an das aufkaufende Unternehmen macht den meist nur kurzzeitigen Arbeitsplatzverlust mehr als wett.

Das einzelne Unternehmen wird durch diesen Wechsel von der Kredit- zur Aktienfinanzierung in eine deutlich turbulentere, weil durch ganz andere Interessen bestimmte Welt versetzt. Auf die Hausbank war Verlass; auf die Fonds ist - nur darauf kann man sich verlassen - kein Verlass. Aus Unternehmenssicht sind die Fonds höchst kapriziöse Partner, von deren guter Laune man unglücklicherweise hochgradig abhängig ist; und gerade wenn man meint, es geschafft zu haben, sie bei bester Laune halten zu können, springen sie ab, weil sie meinen, anderswo noch mehr auf ihre Kosten zu kommen.

Die Investitionsentscheidungen der Fonds sind freilich, genauer betrachtet, hochgradig willkürlich und spiegeln anderen und sich selbst nur vor, rationale Kalkulationen zu sein. Es handelt sich um oftmals extrem gewagte Prognosen der zu- künftigen Gesamtentwicklung eines Unternehmens - im Vergleich zu alternativen Anlagemöglichkeiten - auf der Grundlage seiner vergangenen Performanz. Diese sachliche Maßstabsvergrößerung der Prognoseunsicherheit wird eigentlich fatal da- durch, dass sie durch den sozialen Kontext wechselseitiger Beobachtung zum doppelten Zufall wird. Windolf (2000b, S. 29) führt die Einsicht von John Maynard Keynes an, dass es bei Aktienanlagen nicht einfach darum gehe, wie man selbst ein Unternehmen einschätzt; die eigene Anlage - die ja jeweils nur einen kleinen Teil des benötigten Kapitals bereitstellt - wird vielmehr nur dann profitabel sein, wenn andere Anleger das Unternehmen ähnlich einschätzen und ebenfalls in es investieren. Dies wissen aber alle potentiellen Anleger, weshalb es für jeden - in den Worten von Keynes - um eine Antizipation dessen geht, ,[...] what average opinion expects the average opinion to be." Jeder Anleger muss wissen, was die je anderen Anleger darüber denken, was die je anderen Anleger - zu denen er dann auch immer gehört - denken. Dies ist ein logisch unauflösbarer Zirkel: Wenn jeder seine Entscheidung $(\leftarrow \mathbf{p . 5 3})$ von den Entscheidungen der anderen abhängig macht, kann nie einer den ersten Schritt tun. 
Psycho- und soziologisch wird freilich Entscheidungsfähigkeit hergestellt. Rationalitätsfiktionen springen ein, wo Rationalität schlechterdings unmöglich ist (Schimank 2005, S. 372-393). Zum einen liefern Analysten und Rating-Agenturen Wissenssurrogate über den Zustand eines Unternehmens und seiner Marktchancen; da- ran anknüpfend kann dann auf seiten der Anleger - in situativen Mischungsverhältnissen - einerseits ein Herdentrieb wirksam, andererseits die bewusste Abweichung von dem, was ,alle“ tun, kultiviert werden. Das Aggregatergebnis dieses Zusammenspiels von Beeinflussung der Anleger durch Expertenempfehlungen und wechselseitiger Beobachtung der Anleger untereinander bleibt freilich irreduzibel turbulent, weil es permanent durch heterogenste Informationsschnipsel, die weder hinsichtlich ihrer Verlässlichkeit noch hinsichtlich ihrer Relevanz beurteilt werden können, gefüttert wird und irritiert werden kann.

Natürlich können sich die Aktienwerte nicht völlig von dem loslösen, was ein Unternehmen tatsächlich auf dem Markt leistet und an Profiten einfährt: „Die spekulativen Pirouetten auf den Kapitalmärkten können sich nicht unendlich verselb- ständigen [...]“ (Deutschmann 2005, S. 71) Investment-Fonds können sich keine

„Welt als Wille und Vorstellung“ konstruieren; aber ein ganzes Stück weit und eine ganze Zeitlang - in fallweise variierendem und jeweils unbekanntem Ausmaß - können sich selbst erfüllende Prophezeiungen durchaus wirkmächtig sein, was die Anlageentscheidungen nochmals verkompliziert. Ein Anleger hat $\mathrm{zu}$ all dem schon Ge- sagten hinzukommend auch noch einzukalkulieren, welche realitätssetzende Kraft den Entscheidungen einzelner Anleger - etwa der berühmten Finanzmarkt-Gurus - und den Anlageentscheidungen im Aggregat zukommt. Dann kann es durchaus so sein, dass der Anleger eine Entscheidung trifft, von der er weiß, dass sie sachlich falsch liegt - weil er annimmt, dass wichtige andere Anleger sich so entscheiden wer- den und dies die Verhältnisse gleichsam zurechtrückt. Dennoch: ,[...] es gibt einen Punkt, an dem die auf den Produktmärkten tatsächlich erzielten Renditen unmissverständlich sich geltend machen.“ (Deutschmann 2005, S. 71) Dem Investment- Fond kann es freilich genügen, bis dahin, wenn die spekulative Blase platzt, seinen Gewinn und sich selbst aus dem Staub gemacht zu haben.

III. Das Dilemma der Anleger

Auch wenn die Finanzmärkte letzten Endes an die „Realökonomie“ (Windolf 2005b, S. 25) angebunden bleiben, können sie diese zwischenzeitlich in erheblichem Maße zum Spielball machen, so dass die 
wirtschaftlichen Verhältnisse nicht nur für jedes einzelne von Investment-Fonds dirigierte Unternehmen, sondern ins- gesamt turbulent werden - und damit aufgrund der wirtschaftlichen Verflechtungen auch für selbst nicht auf dem Aktienmarkt gehandelte Eigentümerunternehmen $(\leftarrow \mathbf{p . 5 4})$ gleich welcher Größe, von Oetker bis zum Malermeister. Spätestens hier stellt sich die Frage: Warum agieren die Fonds so, wie sie es tun - und wer steckt dahinter? Eine Antwort hierauf muss in drei Schritten zum Kern des gesamten Geschehens, gleichsam zum „heart of darkness“ - die Anspielung auf Joseph Conrads Roman sei durchaus vielsagend verstanden - des „Finanzmarkt-Kapitalismus“ vorstoßen.

Erstens kann man sich die Analysten anschauen, die für die Fonds arbeiten. Das bleibt jedoch ziemlich unergiebig, denn es handelt sich bei ihnen nicht etwa- so wie bei Ärzten im Gesundheitswesen oder Professoren im Hochschulsystem - um eine gefestigte Profession, die ihre Standards und Strategien der Entscheidungsfindung relativ unbeirrt durch Einflüsse der Organisation und der Klienten, für die sie tätig sind, verfolgen und so dem Geschehen ihren Stempel aufdrücken. Die Analysten sind keine treibende Kraft des „Finanzmarkt-Kapitalismus“, sondern Getriebene. Das zeigt sich nicht zuletzt daran, dass insbesondere ein opportunistischer Optimismus ihren persönlichen Berufserfolg ausmacht: „Analysten, die höhere Gewinne prognostizieren als die tatsächlich erwirtschafteten, haben eine größere Chance, von einem Top-Wertpapierhaus beschäftigt zu werden.“ (Windolf 2005b, S. 42) Wer also im persönlichen Auftreten überzeugend das Blaue vom Himmel verspricht, wird genommen - schon das ist ein Indiz für die insgeheime Verzweiflung der Fonds-Gesellschaften. Man weiß, dass man pures Kaffeesatzlesen betreibt, was man durch

energisch zur Schau getragenen Zweckoptimismus zu überspielen hat. ${ }^{7}$

Wenn also Analysten nicht viel mehr als ausführende Organe des sie beschäftigenden Investment-Fonds sind, kann man sich zweitens der Marktsituation der Fonds zuwenden. Dabei wird man schnell gewahr, dass sie einem knallharten Konkurrenzdruck unterliegen: „Die Konkurrenz um die Gunst des Anlegerpublikums treibt sie dazu, ihre Renditeversprechen in die Höhe zu schrauben. Dadurch werden die Fondsmanager ihrerseits zu Getriebenen in einer selbsterzeugten Dynamik, die der Logik eines Kettenbriefsystems nicht unähnlich ist.“ (Deutschmann 2005,

S. 67) Irgendwann beißen den Letzten die Hunde. Wie bei der Reise nach Jerusalem fällt nicht auf, dass ein Stuhl zu wenig am Tisch steht, solange alle in Bewegung sind; doch wenn es ans Hinsetzen geht, bleibt mindestens einer dumm stehen. Die Vorahnung dieses dicken Endes ist ein weiterer Grund für die innere Verzweiflung der Fonds-Gesellschaften.

Damit kommt man drittens schließlich auf diejenigen zu sprechen, die den Investment-Fonds ihr Geld anvertrauen: die individuellen Anleger. Sie verfahren mit den Fonds nicht anders, als diese mit den

\footnotetext{
${ }^{7}$ Solange man freilich prächtig verdient, handelt es sich um eine höchst fidele Verzweiflung.
} 
Unternehmen: „Die Performanz der Investment-Fonds ist periodisch genau messbar, und sie laufen Gefahr, die Einlagen ihrer Kunden zu verlieren, wenn sie in der Konkurrenz um Profitmaximierung zurückfallen.“ (Windolf 2005b, S. 23) Genau das erzeugt den demonstrativen Optimismus der Versprechungen, in dem die Fonds einander gegenseitig zu überbieten trachten. Wider besseres Wissen muss jeder Fond ein noch rosigeres Bild seiner $(\leftarrow \mathbf{p . 5 5})$ Geldanlagen zeichnen, als es die Konkurrenz tut. Wenn aber diese Eskalation der Versprechungen funktioniert, die Einwerbung des Kapitals der Anleger also gelingt, müssen die Fonds möglichst profitable Anlagemöglichkeiten finden, um die Kunden zumindest hinhalten zu können. Die Fonds agieren ja durchaus ähnlich wie das in Deutschland inzwischen gerichtsnotorische Geldtransportunternehmen Heros, das mehrere hundert Millionen Euro nach folgendem einfachen Rezept veruntreute: Das gestern irgendwo abtransportierte Geld kann auf die Weise unbemerkt in die eigenen Taschen abgezweigt werden, dass heute an dessen eigentlichen Bestimmungsort Geld abgeliefert wird, das kurz zuvor anderswo abgeholt wurde, u.s.w. Genau aus der Besorgnis, dass dieser Verschiebebahnhof nicht auffliegt, rührt der, ,neoliberale“ Furor des politischen Auftretens der Fonds, etwa mit Forderungen nach der schnellen Privatisierung staatlicher Dienstleistungen oder der Öffnung von Märkten wie China für ausländische Anleger.

Was treibt aber die individuellen Anleger, also jenen Teil der Bevölkerung, der zumindest soviel verdient, dass er einen Teil seines Einkommens in Geldanlagen investieren kann? Auf der einen Seite geht es darum, für die aktuelle Lebensführung nicht benötigtes Geld so anzulegen, dass kurz- oder mittelfristig ein Zusatzeinkommen aus Anlagegewinnen hereinkommt. Mit dem Boom des „,neuen Marktes“ der Technologie-Firmen und der Privatisierung der Deutschen Bundespost stiegen auch in Deutschland zahlreiche Kleinaktionäre erstmals ein. Auf der anderen Seite nehmen immer mehr Menschen - auch die, die es sich gar nicht leisten können - ihre Lage so wahr, dass sie zur privaten Alterssicherung Geld anlegen müssen, weil die staatliche Rentenversicherung in einigen Jahren bestenfalls noch eine magere Existenzsicherung werde bieten können. In Deutschland mit seiner hausgemachten demographischen Problematik leuchtet dies besonders leicht ein. In Ländern wie den USA, wo die staatliche Alterssicherung seit jeher viel weniger ausgebaut gewesen ist und u.a. betriebliche Rentenkassen bei vielen Großunternehmen verbreitet gewesen sind, waren dies frühe Einsteiger in den Aktienmarkt. Ein propagandistisch unter- stütztes Bild des „Finanzmarkt-Kapitalismus“ als Emanzipation des Einzelnen zur selbstbestimmten Geldanlage - wider die Zwangsabgabe an eine geldfressende Sozialstaats-Bürokratie und im Vertrauen auf smarte und das Letzte aus dem Markt herausholende Fonds - unterstreicht diese sich verbreitende Sicht der Dinge.

Die Redeweise vom „Volkskapitalismus“, der ursprünglich etwas anders gemeint war, in Gestalt einer „Demokratisierung der Börse“ (Deutschmann 2006b, S. 9) ist natürlich demagogisch - aber als Markie- 
rung eines deutlichen Inklusionsschubs der Bevölkerung in den zumeist über Investment-Fonds realisierten Besitz an Produktionsmitteln mag das Wort durchgehen. Windolf (2005a, S. 16) macht darauf aufmerksam, dass dabei von einer Vermögensumverteilung keine Rede sein kann: „Der Finanzmarkt-Kapitalismus scheint nicht die Kleinaktionäre zu bereichern, sondern führt eher zu exorbitanten Einkommenssteigerungen der Top-Manager." Deutschmann (2005, S. 62) verweist auf der gleichen Linie darauf, dass es unter den Anlegern die Zwei-Klassen-Gesellschaft derer gibt, die ihr Geld exklusiven „Spezial- ( $\leftarrow$ p.56) fonds“ mit sehr hohen Mindestanlagesummen überlassen, und des großen Rests der Anleger, für die nur die „Publikumsfonds“ offen sind - wobei die „Spezialfonds“ über weit mehr Kapital gebieten. Ungeachtet dessen haben jedoch die „Publikumsfonds“ als Tempomacher des „Finanzmarkt-Kapitalismus“ gewirkt. Ihren Rendite- versprechen haben sich die „Spezialfonds“ anpassen müssen, weil sonst das Kapital von ihnen abgewandert wäre. Denn man darf unter den Reichen keine realistischeren Einschätzungen des Möglichen - nicht weniger Geldgier - voraussetzen als bei den Kleinanlegern. Und so gilt eben doch, dass die Haupt-Triebkraft des „Finanz- markt-Kapitalismus“ aus der gesellschaftlichen Mitte kommt.

Die Anlagestrategie dieser Bevölkerungsgruppe ist es also, die näher zu betrachten ist. Diese Strategie spiegelt sich in der bereits geschilderten Logik der Profiterzielung wider, die die Investment-Fonds an den Tag legen: kurzfristige Maximierung des shareholder value als „take the money and run“ von einer Anlage zur nächsten. Dahinter steht erst einmal die von den Medien und den Fonds spätestens in den 1990er Jahren massiv aufgebaute Erwartung, dass schnelle Gewinne in großem Maßstab und dauerhaft möglich sind. Nur mit derartigen Versprechungen hat man die Anleger aus traditionellen Formen der Vermögensbildung - von Sparbüchern bis zur Immobilie - heraus und in den Finanzmarkt hinein locken können. Man hat erst an die Geldgier appelliert, und schnell dann auch an den Zwang, in begrenzter Zeit bis zur Verrentung möglichst viel aus der Geldanlage machen zu müssen. Paradoxer- weise sind diese Appelle angesichts drastischer Enttäuschungen mit dem Zusammenbruch des „,neuen Marktes“ und weiteren Finanzmarktkrisen nicht etwa weniger plausibel geworden. Ganz im Gegenteil haben diejenigen, die erst einmal Misserfolge einstecken mussten, dann erst recht, sozusagen mit dem Übermut der Verzweiflung, darauf gedrängt, diese Verluste mit künftigen Anlagen schnell auszugleichen, um endlich in die Gewinnzone zu wechseln und - in verkürzter Zeit - das ursprünglich Erwartete herausholen zu können. Jedenfalls sind die Fonds ihre vollmundigen, von Anfang an hoch einsteigenden und einander gegenseitig immer noch überbietenden Anfangsversprechungen nicht mehr los geworden - und warum sollte man sie nicht beim Wort nehmen, insbesondere wenn sie einem das versprechen, was man will und vor allem braucht! Nicht wenige Anleger beanspruchen mittlerweile geradezu ein ,'Naturrecht' auf Rendite“ - so weit geht der „Realitätsverlust“ (Deutschmann 2006a, S. 9).

Wendet man den Blick nun auf die größeren gesellschaftlichen Effekte, die der 
„Finanzmarkt-Kapitalismus“ zeitigt, erkennt man, dass sich die gesellschaftliche Mitte nicht nur hinsichtlich ihrer Anlagerenditen täuscht, sondern darüber hinaus und viel unmittelbarer auch hinsichtlich ihrer Arbeitsmarktchancen und sozialstaatlichen Absicherungen selbst ein Bein stellt. Denn das Auftreten der Investment- Fonds wirkt darauf hin,

dass die Arbeitsplätze in den Unternehmen sowie die Aussichten der öffentlich Bediensteten und Selbständigen trotz „Lohnsenkungen und Steigerung der Leistungsanforderungen“ (Deutschmann 2005, S.

77) prekärer werden, $(\leftarrow \mathbf{p . 5 7})$

dass die gesamtwirtschaftliche Arbeitsmarktlage sich selbst in Phasen des Wirtschaftswachstums nicht verbessert

und dass ein internationales „race to the bottom“ hinsichtlich sozialstaatlicher Standards stattfindet, um Investoren anzulocken - bei gleichzeitigem „Subventions- und Steuersenkungswettlauf“ (Deutschmann 2005, S. 79).

Zusammengefasst: Die gesellschaftliche Mitte sorgt für ihre eigene „Prekarität“, weil sie aus den Unternehmen, in denen sie selbst zu einem großen Teil arbeitet bzw. von deren Steuerzahlungen sie als Staatsbediensteter oder Transferempfänger abhängt, das kurzfristige Maximum an Renditen herausholen will. Für jeden einzelnen gilt, dass er - vermittelt über die Investment-Fonds - anderen in gleicher Lage auf die Füße tritt. Manchmal tritt er sogar, ohne es zu merken, sich selbst auf die Füße:

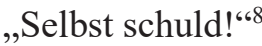

Der Einzelne befindet sich in einem Entscheidungs-Dilemma: Als Arbeitnehmer und analog als Selbständiger - ist er an der Sicherheit seines Arbeitsplatzes, seines Einkommens und seiner Arbeitsbedingungen interessiert; doch als Kapitalanleger will er aus einem Unternehmen an Rendite herausholen, was herauszuholen ist - auf Kosten von Arbeitsplatzsicherheit, Einkommen und Arbeitsbedingungen der dort Beschäftigten und auf Kosten der Steuerzahlungen dieses Unternehmens. Deshalb gründet sich der „Finanzmarkt-Kapitalismus“ - wie Deutschmann (2005, S. 81) festhält - „,[...] auf den Konsens der politisch tonangebenden oberen Mittelschichten, gerade auch der mit Hilfe des sozialdemokratischen Wohlfahrtsstaates Aufgestiegenen.“ Und er zitiert Hans Peter Martin und Harald Schumann: „Der gut bezahlte Mittelstandsbürger ist daher nur zu oft Opfer und Täter, Gewinner und Verlierer zugleich." Der einzelne Anleger steckt also, spieltheoretisch betrachtet, in einem prisoner's dilemma: Für jeden Anleger wäre es das beste, er brächte sich und die anderen nicht in die Bredouille, den Eigennutz maximieren zu müssen - doch weil keiner mit einer entsprechenden Zurückhaltung der anderen rechnen kann, muss jeder die Maximierung des Eigennutzes betreiben, was allen schadet.

\footnotetext{
${ }^{8}$ So der Titel eines Artikels in DIE ZEIT 24/2006, in dem es um das analoge Phänomen geht: „Ob Niedriglöhne, Stellenabbau oder Umweltzerstörung: Was uns als Bürger em- pört, fördern wir als Kunden.“
} 
Diese allseitige Maximierungsneigung wird durch zwei schon angesprochene Randbedingungen noch angestachelt. Zum einen konkurrieren die Fonds untereinander und versprechen den Anlegern allesamt unrealistische Renditen. Zum anderen hat sich die staatliche Wirtschafts- und Sozialpolitik parallel - und flankierend - zum Aufkommen des „Finanzmarkt-Kapitalismus“ so gewandelt, dass Sozialleistun- gen ebenso wie Arbeitnehmerrechte, etwa beim Kündigungsschutz, abgebaut wer- den, um in der internationalen Standortkonkurrenz ein ,investitionsfreundliches Klima“ präsentieren zu können. Wie die unsicher gewordene staatliche Rentenversicherung treiben auch diese Entwicklungen den einzelnen Arbeitnehmer an, wenn er irgend kann, „Eigenvorsorge“ zu betreiben. Denn das zunehmend sichtbar gewordene $(\leftarrow \mathbf{p . 5 8})$ „Prekariat“ führt ihm vor, dass es ihm auch so ergehen könnte. Die Jagd nach schnellen hohen Renditen hält so an, wodurch alle Anleger einander wechselseitig als Arbeitnehmer nur noch immer mehr gefährden, u.s.w.

$I V$. Wieder nur frei erfunden?

So also darf man sich den „Finanzmarkt-Kapitalismus“ gesellschaftstheoretisch denken: als eine abermals mit der Selbstabschaffung einer gesellschaftlichen Gruppe ein- hergehende radikale Krise des Kapitalismus. Insoweit handelt es sich um die vertraute Denkfigur.

Aber auch Unterschiede sind deutlich geworden. Heute geht es nicht um die Kapitalisten, sondern um die gesellschaftliche Mitte als historisches Subjekt. Und es geht heute nicht mehr um die Abschaffung des Kapitalismus, sondern - so ist zu vermuten - um einen drohenden Rückfall in quasi frühkapitalistische Verhältnisse: Nach einiger Zeit stehen einige wenige Glücksritter vielen armen Teufeln gegenüber, die entsprechend leicht ausbeutbar sind, sich also auch kaum mehr aus eigener Kraft in gesicherte Lebensverhältnisse hinüber retten können. Ob in dieser unbarmherzigen „Erfolgsgesellschaft“ (Neckel 2001) dann Marx’ ursprüngliche Prognose doch noch zutrifft und die Zuspitzung sozialer Not den Kapitalismus explodieren lässt, oder ob der Sprengsatz wodurch auch immer - vielleicht durch ein minimal kalkuliertes garantiertes „Grundeinkommen“, man könnte auch von „Armengeld“ sprechen, wird aber ganz sicher eine freundlichere Formulierung finden - wieder einmal entschärft wird, muss hier dahingestellt bleiben. ${ }^{9}$ Gäbe es eine Revolution, hätte

\footnotetext{
${ }^{9}$ Die Theoretiker des „Finanzkapitals“ der 1920er Jahre waren in der Frage, wie sich der Un- tergang des Kapitalismus vollziehen würde, ebenso optimistisch wie die Stamokap-Theore- tiker der 1960er Jahre: Die kapitalistischen Großunternehmen tasten sich, ohne es zu mer- ken, durch Größenwachstum, Monopolisierung und entsprechende interne Planungser- fordernisse und Abstimmungen untereinander, etwa in Form von Kartellen, an eine sozia- listische Planwirtschaft heran. Nach der Revolution braucht man dann nur noch die Ma- nager durch Planifikateure zu ersetzen. So gemütlich sieht das jetzige Szenario des „Finanzmarkt-Kapitalismus" nicht aus.
} 
Marx seine Rechnung letzten Endes nur in dem Sinne zu einfach kalkuliert, dass es genau jener Bevölkerungsgruppe, von der er annahm, sie würde zwischen den Polen von Kapitalisten und Arbeitern zermahlen, noch bevor diese final aufeinander losgehen, bedurfte, um als „Totengräber“ des Kapitalismus zu fungieren. Nicht das Proletariat, erst die aus der ,affluent society“, ihrem anfänglichen Erfolg wie ihrem letztendlichen Scheitern hervorgegangene, durch Aktienanlagen vollzogene Inklusion von vielen Menschen aus der gesellschaftlichen Mitte in die Gruppe der Besitzer von Produktionsmitteln hätte dann jene Munition gebündelt, durch die endgültig alles

„,in die Luft gesprengt wird“ (Marx/Engels 1848, S. 56).

Hier ging es, wie gesagt, lediglich darum, einen analytischen Vorschlag auszubuchstabieren, wie sich der gegenwärtige „Finanzmarkt-Kapitalismus“ fassen lässt, $(\leftarrow$ p.59) und die Ähnlichkeit der prinzipiellen Denkfigurzurklassischen Kapitalismusanalyse von Marx zu akzentuieren. Ob wir es auch dieses Mal nur mit einer fesselnden, aber zum Glück frei erfundenen Story zu tun haben, wird sich erweisen. Wir sollten uns da nicht zu sicher sein. $(\leftarrow \mathbf{p . 6 0})$

\section{Literatur}

Bourdieu, Pierre et al., 1993: Das Elend der Welt. Zeugnisse und Diagnosen alltäglichen Lei- dens an der Gesellschaft, Konstanz 1997: Universitäts-Verlag Konstanz.

Bourdieu, Pierre, 1998: Gegenfeuer. Wortmeldungen im Dienste des Widerstands gegen die neo-liberale Invasion, Konstanz: Universitäts-Verlag Konstanz.

Brödner, Peter, 1985: Fabrik 2000. Alternative Entwicklungspfade in die Zukunft der Fabrik, Berlin: Sigma.

Bude, Heinz, 1998: Die Überflüssigen als transversale Kategorie, in: Peter A. Berger und Mi- chael Vester (Hrsg.), Alte Ungleichheiten, neue Spaltungen, Opladen: Leske + Budrich.

Coupland, Douglas, 1991: Generation X - Geschichten für eine immer schneller werdende Kultur, München: Goldmann.

Dahrendorf, Ralf, 1992: Der moderne soziale Konflikt. Essay zur Politik der Freiheit, Stuttgart: Deutsche Verlags-Anstalt.

Deutschmann, Christoph, 2005: Finanzmarkt-Kapitalismus und Wachstumskrise, in: Paul Windolf (Hrsg.), Finanzmarkt-Kapitalismus. Analysen zum Wandel von Produktionsregi- men. Sonderheft 45 der Kölner Zeitschrift für Soziologie und Sozialpsychologie, Wiesba- den: VS, S. 58-84.

Deutschmann, Christoph, 2006a: Die Herrschaft der Rentiers. Finanzmarkt-Kapitalismus und politische Demokratie. Ms. 
Deutschmann, Christoph, 2006b: Keynes und der Finanzmarkt-Kapitalismus. Ms. Heimann, Eduard, 1929: Soziale Theorie des Kapitalismus - Theorie der Sozialpolitik, Frank-

furt a.M., 1980: Suhrkamp.

Martin Höpner und Lothar Krempel, 2004: The Politics of the German Company Network, in: Competition and Change 8, S. 339-356.

Galbraith, John Kenneth, 1958: The Affluent Society, Harmondsworth, 1979: Penguin. Klundt, Michael, 2005: ,Saving capitalism from itself"? Entstehung und Entwicklung sozialdemokratischer Wohlfahrtsstaatlichkeit, in: Karl Gabriel (Hrsg.), Europäische Wohlfahrts- staatlichkeit Soziokulturelle Grundlagen und religiöse Wurzeln. Jahrbuch für Christliche Sozialwissenschaften 46/2005, S. 129-146.

Kronauer, Martin, 1998: „Exklusion“ in der Systemtheorie und in der Armutsforschung. An- merkungen zu einer problematischen Beziehung, in: Zeitschrift für Sozialreform 44, S. 755-768.

Luhmann, Niklas, 1994: Inklusion und Exklusion, in: Niklas Luhmann, Soziologische Aufklä- rung, Bd. 6. Die Soziologie und der Mensch, Opladen 1995: Westdeutscher Verlag, S. 234-267.

Marcuse, Herbert, 1964: Der eindimensionale Mensch, Neuwied/Berlin 1972: Luchterhand. Marx, Karl, 1867: Das Kapital. Erster Band, Berlin 1965: Dietz.

Marx, Karl, 1894: Das Kapital. Dritter Band, Berlin 1979: Dietz.

Marx, Karl und Friedrich Engels, 1848: Manifest der Kommunistischen Partei, Berlin 1945: Dietz.

Merton, Robert K., 1936: The Unanticipated Consequences of Purposive Social Action, in: American Sociological Review 1, S. 894-904.

Neckel, Sighard, 2001: „Leistung“ und „Erfolg“. Die symbolische Ordnung der Marktgesell- schaft, in: Eva Barlösius, Hans-Peter Müller und Steffen Sigmund (Hrsg.), Gesellschaftsbil- der im Umbruch. Soziologische Perspektiven in Deutschland, Opladen: Leske \& Budrich, S. 245-265.

Schimank, Uwe, 2005: Die Entscheidungsgesellschaft. Komplexität und Rationalität der Mo- derne, Wiesbaden: VS.

Schultheis, Franz und Kristina Schulz (Hrsg.), 2005: Gesellschaft mit begrenzter Haftung - Zumutung und Leiden im deutschen Alltag, Konstanz: Universitätsverlag Konstanz.

Windolf, Paul, 2005a: Die neuen Eigentümer, in: Paul Windolf (Hrsg.), Finanzmarkt-Kapita- lismus. Analysen zum Wandel von Produktionsregimen. Sonderheft 45 der Kölner Zeit- schrift für Soziologie und Sozialpsychologie, Wiesbaden: VS, S. 8-19.

Windolf, Paul, 2005b: Was ist Finanzmarkt-Kapitalismus?, in: Paul Windolf (Hrsg.), Finanz- markt-Kapitalismus. Analysen zum Wandel von Produktionsregimen. Sonderheft 45 der Kölner Zeitschrift für Soziologie und Sozialpsychologie, Wiesbaden: VS, S. 20-57. 